Case report

\title{
Ovarian Cancer That Was Initially Diagnosed as Malignant Pleural Effusion of Unknown Primary Origin
}

\author{
Toshihiko Fukuoka, Eisuke Matsuoka, Sahoko Chiba, Satoshi Takayama and Satoshi Ohno \\ Department of Respiratory Medicine, Toride Kyodo General Hospital, Ibaraki, Japan
}

\begin{abstract}
We report a case of adenocarcinoma detected in the right pleural effusion of a 75-year-old woman. Investigations failed to reveal the site of the primary lesion, and the case was treated as primary unknown cancer. The pleural effusion disappeared after chemotherapy; however, as there was serious bone marrow suppression, the clinical course was observed at an outpatient clinic without chemotherapy. A search for the primary lesion was repeated, but it was not found. One year after first admission, a chest X-ray showed left pleural effusion. Adenocarcinoma was detected in the effusion and a tumor mass obtained from the pleural cavity. Ovarian cancer was diagnosed based on the histological, serological and MRI findings. Thus, this was a rare case of ovarian cancer in which the diagnosis was confirmed by repeated evaluation and in which the initial diagnosis had been primary unknown cancer with malignant pleural effusion only.
\end{abstract}

Key words: ovarian cancer, primary unknown cancer, malignant pleural effusion

(J Rural Med 2009; 4(1): 41-44)

\section{Introduction}

Ovarian cancer with malignant pleural effusion is often associated with ascites ${ }^{1}$. Cases of ovarian cancer with malignant pleural effusion only are rare $^{2)}$, and there have been few cases of ovarian cancer that have initially been categorized as primary unknown cancer with malignant pleural effusion only, ${ }^{3,4}$.

We report a rare case of ovarian cancer in which the diagnosis was confirmed by repeated evaluation and in which the initial diagnosis had been primary unknown cancer with malignant pleural effusion.

Correspondence to: Toshihiko Fukuoka

Department of Respiratory Medicine, Toride Kyodo General Hospital, 2-1-1 Hongo, Toride City, Ibaraki 302-0022, Japan

E-mail: torikyo@medical.email.ne.jp

\section{Case Report}

A 75-year-old woman was admitted to our hospital complaining of right chest pain and dyspnea on effort (HughJones II-III). Respiratory sound was reduced at the right lung on auscultation. Chest X-ray showed right pleural effusion (Fig. 1).

The results of routine laboratory examinations were within normal limits. Cytology for pleural effusion revealed Class V adenocarcinoma (Fig. 2). Serological exam revealed a mildly elevated level of CA125 (44.5 IU/L: normal range was less than 35 ).

We searched for the origin of the cancer, but were unable to detect the origin on chest and abdominal CT (ascites was

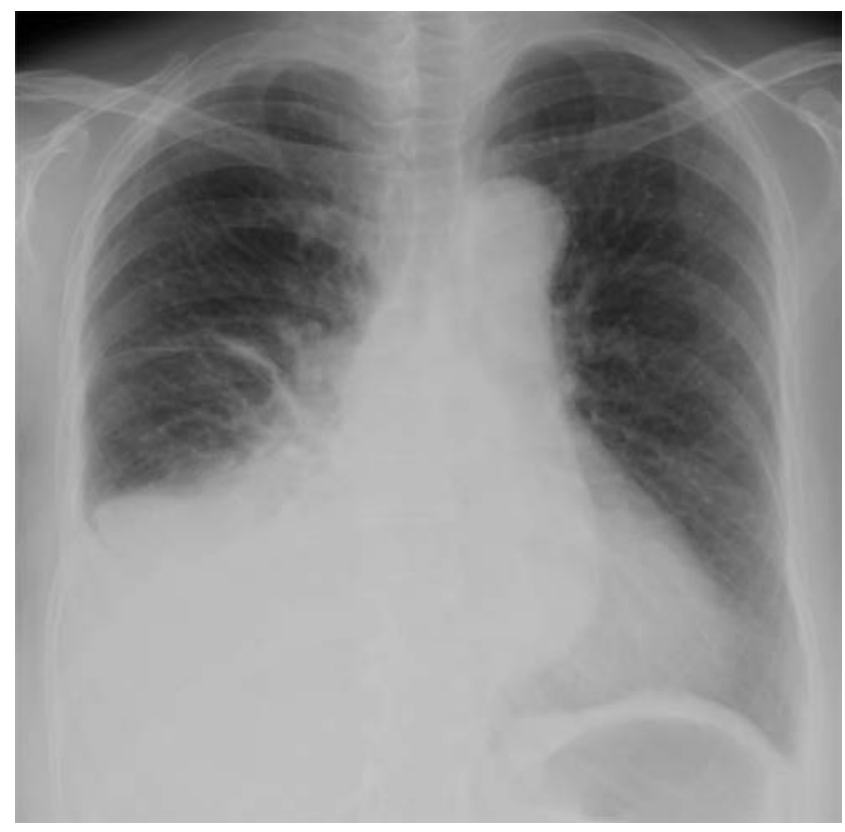

Figure 1 Chest X-ray on admission. Right pleural effusion was detected. 


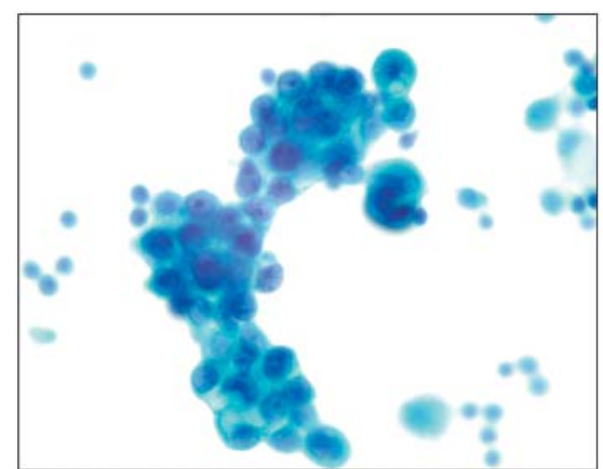

Papanicolaou $x 400$

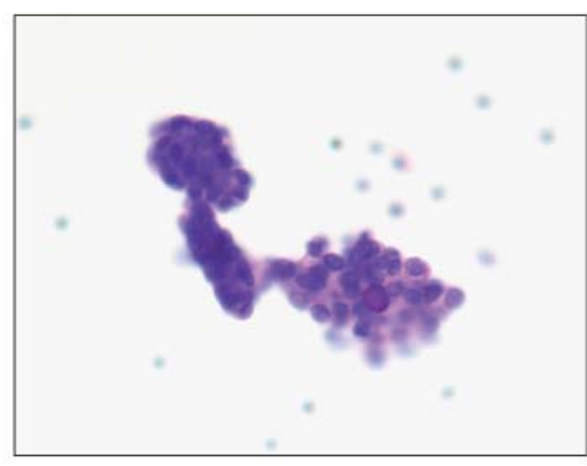

PAS $\times 20$

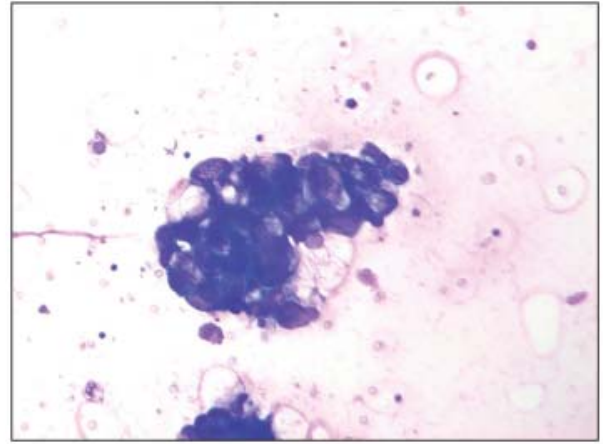

Giemsa $\times 40$

Figure 2 Cytology for pleural effusion. Giemsa, PAS and Papanicolaou's stain.

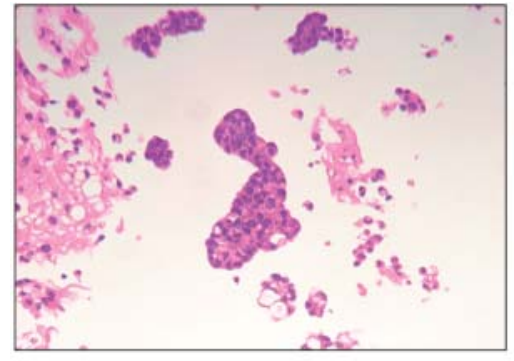

HE $\times 20$

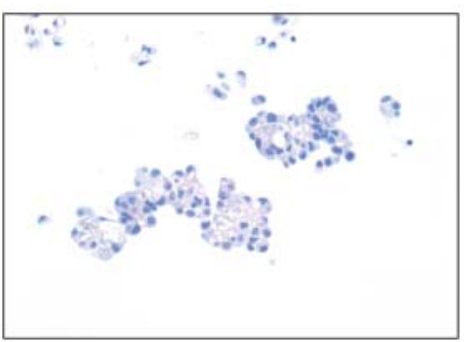

CEA $\times 40$

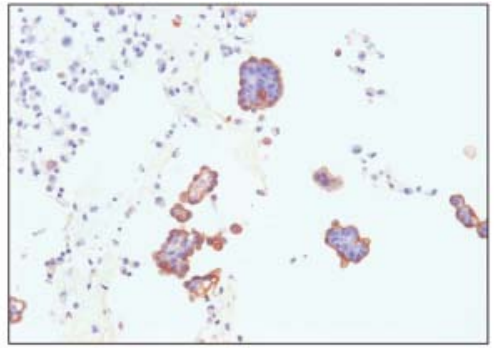

EMA $\times 40$

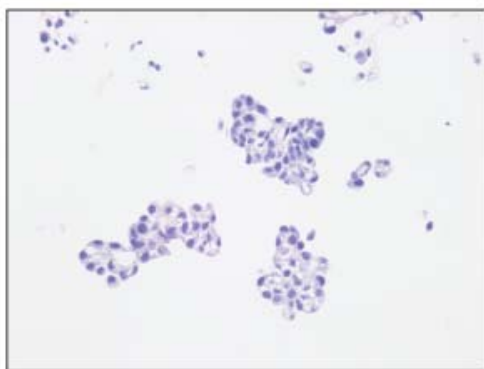

Calretinin $\times 40$

Figure 4 Histology for the mass. Positive for EMA and keratin and negative for calretinin and CEA. 

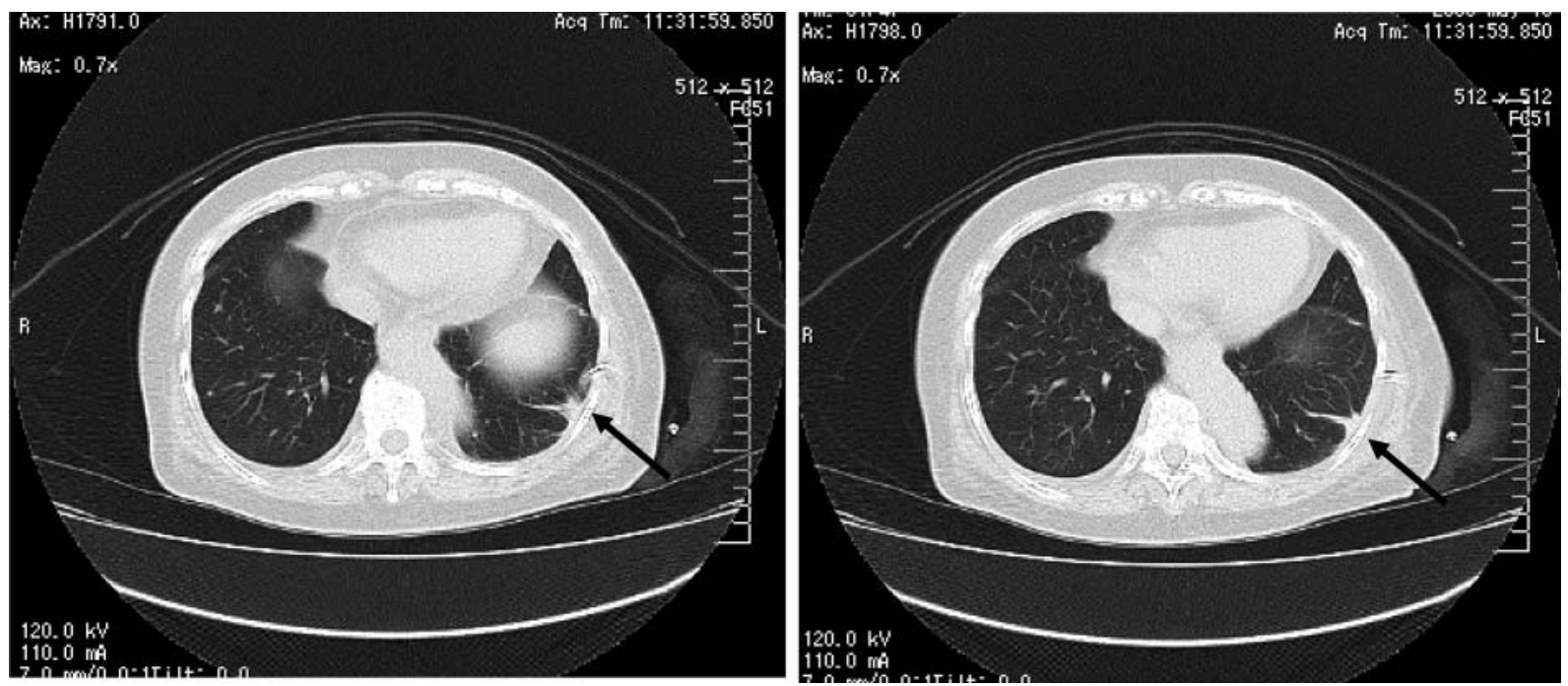

Figure 3 Chest CT scan revealed a nodal shadow in the pleura at the arrow.

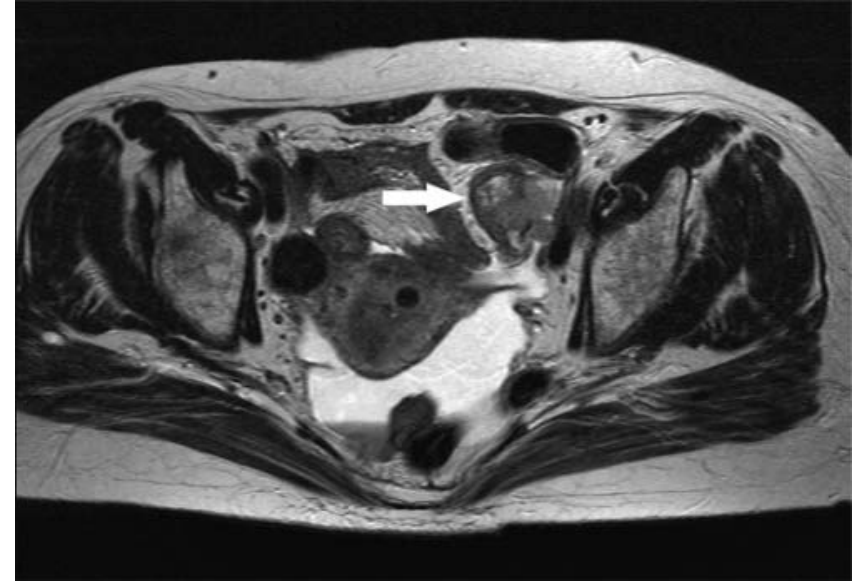

Figure 5 Abdominal MRI. The arrow indicates a nodule detected in the ovary.

not present), thyroid and mammary echocardiography, gastroscopy, or fiberscopic examination of the colon. The patient refused thoracoscopy. Chemotherapy was therefore performed for primary unknown cancer. Two cycles of 600 $\mathrm{mg}$ carboplatin [day 1, dosed at a $5 \mathrm{mg} / \mathrm{mL} / \mathrm{min}$ target AUC (area under the free carboplatin plasma concentration versus time curve)] and $100 \mathrm{mg}$ paclitaxel (days 1, 8, 15) were performed. The pleural effusion disappeared after the chemotherapy; however, bone marrow suppression was quite severe, with grade 4 (National Cancer Institute common toxicity criteria) granulocytopenia, so the chemotherapy was discontinued after 2 cycles. The clinical course was observed in our outpatient clinic. CT and PET examinations were regularly performed on an outpatient basis, but did not reveal the origin of the tumor.

One year after first admission, left pleural effusion was noted. Chest CT revealed a nodal shadow in the pleura (Fig. 3 ), and ascites was noted. A tumor mass was excised via a tube inserted into the pleural cavity. Histologically, the mass showed papillary projections. Immunohistological study of the mass, which was positive for EMA and keratin and negative for calretinin and CEA, suggested ovarian carcinoma (Fig. 4). Serological exam revealed a highly elevated level of CA125 (2412 IU/L). Abdominal MRI revealed a nodal shadow $(3 \times 4 \mathrm{~cm})$ in the left ovary (Fig. 5). Fiberscopic examination of the colon revealed no mass in that area.

Based on the histological findings, high CA125 level and nodular shadow in the left ovary on abdominal MRI, ovarian cancer was diagnosed. Thus, the patient was referred to a gynecologist.

\section{Discussion}

This was a case of ovarian cancer in which the diagnosis was confirmed by repeated evaluation and in which the initial diagnosis had been primary unknown carcinoma. Primary unknown cancer accounts for $3 \%$ of all malignant disease, and $80 \%$ of cancers of unknown origin are adenocarcinomas ${ }^{5)}$. The origin is ultimately detected in $10-15 \%$ of $\operatorname{cases}^{3,6)}$. The lung, pancreas, liver and gastrointestinal tract are often the origin, while it is rare for the ovary to be the origin, accounting for about $2-4 \%$ of such cases ${ }^{3}$. An autopsy report noted that 43 of 1656 cases were initially diagnosed as having cancer of unknown origin, 23 of which were confirmed to have 
had adenocarcinoma by autopsy, and none of the cases had ovarian cancer ${ }^{7}$. Among 96 cases with malignant pleural effusion, lung cancer and mammary cancer were most frequently observed, followed by ovarian cancer (in 9 cases) ${ }^{2}$. In addition, $3 \%$ of ovarian cancers are associated with pleural effusion $^{2}$. In 187 female cases of malignant pleural effusion, $15.2 \%$ of the cases had ovarian cancer, and all had ascites ${ }^{1)}$. In another report, 3 of 25 ovarian cancer cases that were initially categorized as extra-abdominal adenocarcinoma of unknown primary origin had malignant pleural effusion ${ }^{4}$. The present case was thus a rare one of ovarian cancer with subsequent malignant pleural effusion and without ascites.

The tumor origin could not be detected by PET examination in our patient. The sensitivity of PET in detection of ovarian cancer is $60 \%$, and its specificity is $70 \%{ }^{8}$. Since PET cannot detect ovarian cancer of less than $1 \mathrm{~cm}$ in diameter ${ }^{9}$, the tumor in our patient was too small to be detected.

The standard chemotherapy for unknown primary carcinoma is a combination of carboplatin and paclitaxe ${ }^{10)}$. Since the standard chemotherapy for ovarian cancer is the same, it was effective in our patient.

This was a case of ovarian cancer in which the diagnosis was confirmed by repeated evaluation and in which the initial diagnosis was unknown primary carcinoma. We searched unsuccessfully for literature that mentioned how malignant cells of the ovary move directly to the pleura. We consider that the malignant cells of the ovary may have moved to pleura directly via the blood stream, leading to production of pleural effusion without producing ascites, making diagnosis difficult at first. It would have been interesting if we could have revealed the biological properties of the malignant cells, but we could not.

Although early diagnosis of ovarian cancer was difficult in our case, diagnosis might have been possible with thoracoscopy. Thus, in cases of unknown primary cancer with malignant pleural effusion only, thoracoscopy should be performed in patients who agree to it.

\section{References}

1) Johnston WW. The malignant pleural effusion. A review of cytopathologic diagnoses of 584 specimens from 472 consecutive patients. Cancer 1985; 56: 905-909.

2) Chernow B, Sahn SA. Carcinomatous involvement of the pleura. An analysis of 96 patients. Am J Med 1977; 63: 695702 .

3) Nystrom JS, Weiner JM, Heffelfinger-Juttner J, Irwin LE, Bateman JR, Wolf RM. Metastatic and histologic presentations in unknown primary cancer. Semin Oncol 1977; 4: 53-58.

4) Rongyu Z, Zhiyi Z, Shumo C. Ovarian carcinoma presents as distant metastases without detectable tumors of the origin disease at the first presentation. Chin J Obstet Gynecol 2001; 36: 414-416 (in Chinese, Abstract in English).

5) Pavlidis N, Briasoulis E, Hainsworth J, Greco FA. Diagnostic and therapeutic management of cancer of an unknown primary. Eur J Cancer 2003; 39: 1990-2005.

6) Shildt RA, Kennedy PS, Chen TT, Athens JW, O'Bryan RM, Balcerzak SP. Management of patients with metastatic adenocarcinoma of unknown origin: A southwest oncology group study. Cancer Treat Rep 1983; 67: 77-79.

7) Mayordomo JI, Guerra JM, Guijarro C, Garcia-Prats MD, Gomez A, Lopez-Brea M, Gonzalez R, Hergueta P, LopezPino MA, Martinez-Tello F, Cortes-Funes H. Neoplasms of unknown primary site: A clinicopathological study of autopsied patients. Tumori 1993; 79: 321-324.

8) Nakamoto Y, Saga T, Fujii S. Positron emission tomography application for gynecologic tumors. Int Gynecol Cancer 2005; 15: 701-709.

9) Yen TC, Lai CH. Positron emission tomography in gynecologic cancer. Semin Nucl Med 2006; 36: 93-104.

10) Briasoulis E, Kalofonos H, Bafaloukos D, Samantas E, Fountzilas G, Xiros N, Skarlos D, Christodoulou C, Kosmidis $\mathrm{P}$, Pavlidis N. Carboplatin plus paclitaxel in unknown primary carcinoma: A phase II Hellenic cooperative oncology group study. J Clin Oncol 2000; 18: 3101-3107. 